\title{
Far-Ultraviolet Observations of Supernova Remnants
}

\author{
By WILLIAM P. B LA IR
}

The Johns Hopkins University, Dept. of Physics and Astronomy, Baltimore, MD 21218, USA

New observations of supernova remnants in the far ultraviolet, especially in the sub-Ly $\alpha$ region, are changing the way we look at the interaction between blast waves and the interstellar medium. I briefly review some of the recent FUV observations of supernova remnants from the Hopkins Ultraviolet Telescope, the Voyager Ultraviolet Spectrometers, as well as IUE and HST.

\section{Introduction}

Observations with the IUE satellite over the last 16 years have permitted great strides to be made in better understanding supernova remnants (SNRs) and their interaction with the interstellar medium (ISM). In particular, many filaments have been observed in the galactic SNRs Vela and the Cygnus Loop (Raymond et al. 1988; Raymond, Wallerstein, \& Balick 1991; Hester, Raymond, \& Blair 1993; and references therein), and a few studies have been made of bright remnants in the Magellanic Clouds (Vancura et al. 1992a; Blair et al. 1989). However, IUE (and even HST) is limited to wavelengths longer than about $1200 \AA$, and it is only in the last few years that significant inroads have been made at FUV wavelengths down to the Lyman limit at $912 \AA$. These observations have been made with the Ultraviolet Spectrometers (UVSs) onboard the Voyager interplanetary spacecraft, and the Hopkins Ultraviolet Telescope (HUT) onboard the Astro-1 space shuttle mission in December 1990. In separate sections below I will discuss some of the recent advances from each of these instruments.

\section{Voyager UVS Results}

The Voyager UVSs are low resolution $(\Delta \lambda \sim 35 \AA$ for extended sources) instruments with large fields of view $\left(0.1^{\circ} \times 0.86^{\circ}\right)$ and no intrinsic spatial resolution capabilities (see Broadfoot et al. 1981). They were, of course, mainly intended to observe the atmospheres of the outer planets, with wavelength coverage from $500-1700 \AA$. Even so, with very low background rates these small spectrographs have been able to detect three galactic SNRs in the sub-Ly $\alpha$ spectral region. These observations show the $912-1200$ $\AA$ spectrum to be dominated by two peaks at 980 and $1035 \AA$, due mainly to C III $\lambda 977$ and $\mathrm{O}$ VI $\lambda \lambda 1032,1038$. These peaks vary in relative intensity from object to object and from place to place within the individual objects, indicating differences in the types and velocities of shocks within the large Voyager FOV at any given position.

For galactic SNRs with large angular size, the Voyager UVSs can provide some crude spatial information by summing individual (short integration) spectra as a function of position on the sky. Blair et al. (1991a) used over 15,000 Voyager spectra to produce FUV "maps" of the Cygnus Loop in C III and O VI, sampling optical and X-ray data in the same manner for comparison. Considerable line intensity variations were detected as a function of position, and a globally-averaged O VI:C III ratio near 2 was found. The luminosity of the Cygnus Loop in O VI was found to be $4.6 \times 10^{36} \mathrm{ergs} \mathrm{s}^{-1}$, roughly 4 times the 0.1-4.5 keV X-ray luminosity reported by $\mathrm{Ku}$ et al. (1984). Rasmussen \& Martin 


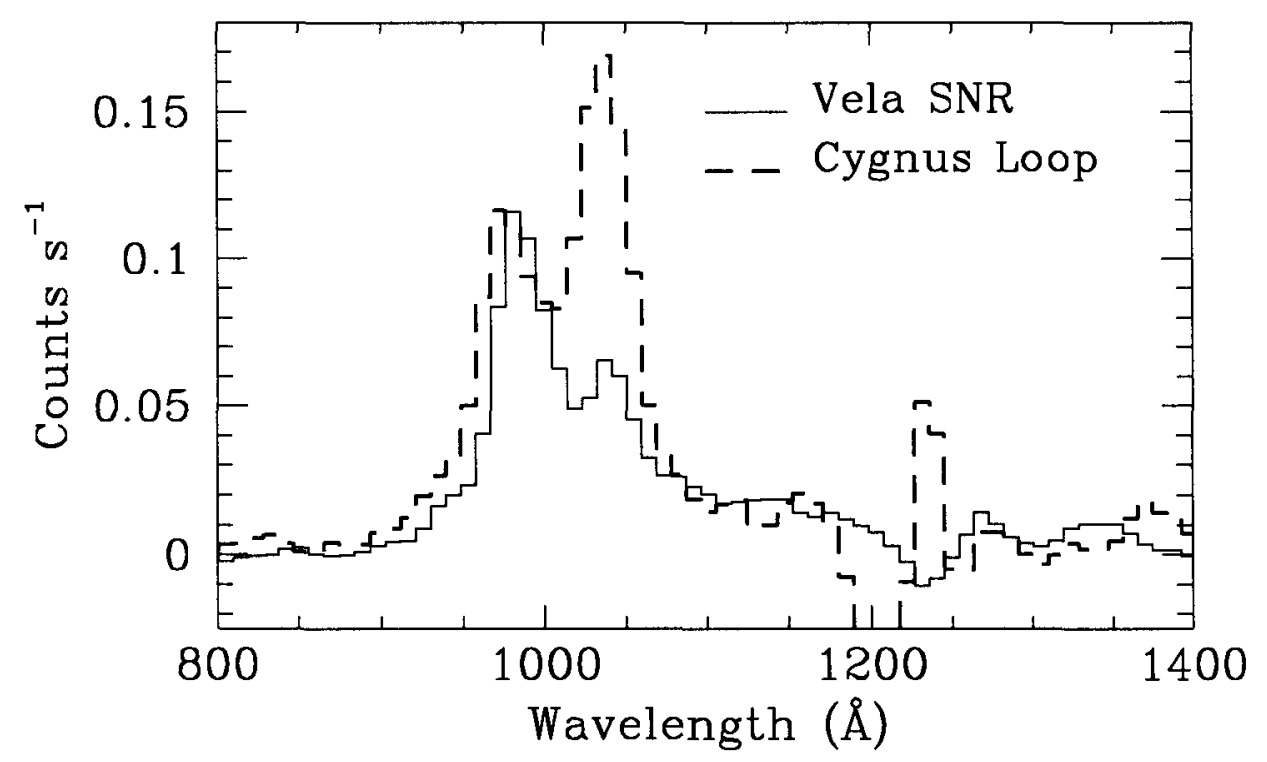

FIGURE 1. A comparison of Voyager UVS spectra of the Vela (solid line) and Cygnus Loop (dashed line ) SNRs. Note the change in relative intensity of the two peaks, which are attributed to C III $\lambda 977$ and O VI $\lambda 1035$.

(1992) found a lower O VI luminosity roughly comparable to the soft X-ray luminosity using a rocket experiment. While reasons for this discrepancy are not understood, it remains clear that the luminosities of individual UV (and by analogy, optical) lines are roughly on a par with the total soft X-ray luminosity. This global perspective of the Cygnus Loop's UV emission was only obtainable with the Voyager spacecraft.

Voyager has also been used to perform more detailed studies of selected regions in the Cygnus Loop. In a recent paper, Vancura et al. (1993) report selected Voyager observations stepping across the main blast wave in the Cygnus Loop. They estimate that the main blast wave accounts for 10-15\% of the total O VI flux observed by Blair et al. (1991a). The remainder arises from radiative shocks more closely associated with the bright optical filaments.

The Vela SNR also has a large angular size and low reddening, making it available to Voyager FUV observations. Blair, Vancura, \& Long (1993) report observation of a filament in the extreme SE portion of Vela that corresponds to a "breakout" from the $\mathrm{X}$-ray shell. The optical filaments at this location are quite reminiscent of Cygnus Loop filaments, appearing to be a large sheet of material viewed approximately edge-on. The Voyager spectrum at this position in Vela is compared with the global Cygnus Loop spectrum in Figure 1.1. The sub-Ly $\alpha$ spectrum of Vela is dominated by the same two peaks seen in the Cygnus Loop, but the relative intensity of O VI is much weaker than in the Cygnus Loop. An ongoing effort to map a section of the northern rim of Vela (Blair et al., in preparation) indicates that this weaker O VI emission is apparently a general feature of Vela and not restricted to the SE filaments. This implies a smaller filling factor of $\geq 160 \mathrm{~km} \mathrm{~s}^{-1}$ shocks in Vela than in the Cygnus Loop.

A third galactic SNR has recently been detected with Voyager. G65.3+5.7 is a large diameter SNR with relatively faint optical and X-ray emission. In particular, the optical filaments are peculiar, consisting of large smooth arcs of emission relatively strong in [O III] $\lambda 5007$ (Fesen, Gull, \& Ketelson 1983). The Voyager spectrum of one of these 
filaments (Fesen \& Blair, in preparation) shows an O VI:C III ratio of unity, intermediate to the Vela and Cygnus Loop results.

Hence, while the strong [O III] optical emission could only indicate the presence of shocks with velocity $\geq 100 \mathrm{~km} \mathrm{~s}^{-1}$, the presence of strong O VI indicates shocks of $\geq 160 \mathrm{~km} \mathrm{~s}^{-1}$ must be occurring in this old SNR.

$\mathrm{X}$-ray data for the Cygnus Loop (Ku et al. 1984) indicate a velocity of $\sim 400 \mathrm{~km} \mathrm{~s}^{-1}$ for the main blast wave, while optical and IUE results have pointed toward shocks with velocities near $100 \mathrm{~km} \mathrm{~s}^{-1}$ in the bright optical filaments (cf. Raymond et al. 1988, and references therein). The presence of strong and ubiquitous $O$ VI emission in these objects provides widespread evidence for shocks with intermediate velocities in excess of $160 \mathrm{~km} \mathrm{~s}^{-1}$ in the bright filamentary structures of galactic SNRs. This may indicate thermally unstable cooling, evaporative processes, or a more extensive intermediate density component of the ISM than has been recognized previously.

\section{HUT Results}

The Hopkins Ultraviolet Telescope was flown on the space shuttle Columbia for 9 days in December 1990 as part of the Astro- 1 mission. HUT consists of a $0.9 \mathrm{~m}$ telescope and a prime focus FUV spectrograph with a microchannel-plate intensifier and reticon readout. The spectrograph provided coverage from $830-1860 \AA$ at about $3 \AA$ resolution. Seven selectable apertures provide some flexibility in matching the needs of individual observations, but no intrinsic spatial resolution was available. Further details on the instrument and its performance can be found in Davidsen et al. (1992). The excellent sensitivity, good resolution, and wavelength coverage of HUT make it an ideal instrument for studying interstellar shocks. Unfortunately, the brevity of the mission meant that only a few key observations of SNRs could be made.

The HUT observations allowed a much more detailed look at individual filaments in the Cygnus Loop (see Figure 1.2). Blair et al. (1991b) used a 9.4" x 116" aperture with HUT to isolate emission from a bright radiative filament in the eastern portion of the remnant. This was the first spectrum to resolve the full sub-Ly $\alpha$ spectrum of an interstellar shock wave, and showed without a doubt that the peaks seen with Voyager corresponded mainly to C III $\lambda 977$ and O VI $\lambda \lambda 1032,1038$, with other lines at a much lower level. The O VI doublet was observed in the $2: 1$ ratio indicative of optically thin conditions, and the $\mathrm{O}$ VI:N V and O VI:C IV ratios were both consistent with the expectations from a $170 \mathrm{~km} \mathrm{~s}^{-1}$ shock wave. However, contrary to the optical appearance of this filament (which indicates extreme incompleteness of the cooling and recombination zone), the intermediate ionization lines in the UV were stronger that predicted by a single, steadyflow shock model. Hence, the possibilities of thermal instabilities (cf. Innes 1992) and/or multiple shock velocities within the aperture were indicated.

Subsequent work on this region of the Cygnus Loop is in progress.

We are using IUE data, longslit optical spectra, and optical imagery to investigate spatial variations within the HUT aperture location. A preliminary analysis of these data indicates that the agreement of the O VI:N V and O VI:C IV ratios with a $170 \mathrm{~km} \mathrm{~s}^{-1}$ may have been fortuitous. Both $\mathrm{N} \mathrm{V}$ and C IV are apparently affected by resonance line scattering at some positions along the HUT aperture (an effect also indicated by UV imaging observations from Astro-1, see Cornett et al. 1992). Multiple shock velocities are almost certainly present, and the peak velocity in the HUT aperture may be considerably higher than the previous estimate of $170 \mathrm{~km} \mathrm{~s}^{-1}$.

A second position observed by HUT was the "nonradiative" filament in the NE Cygnus Loop previously observed with IUE by Raymond et al. (1983). Long et al. (1992) 


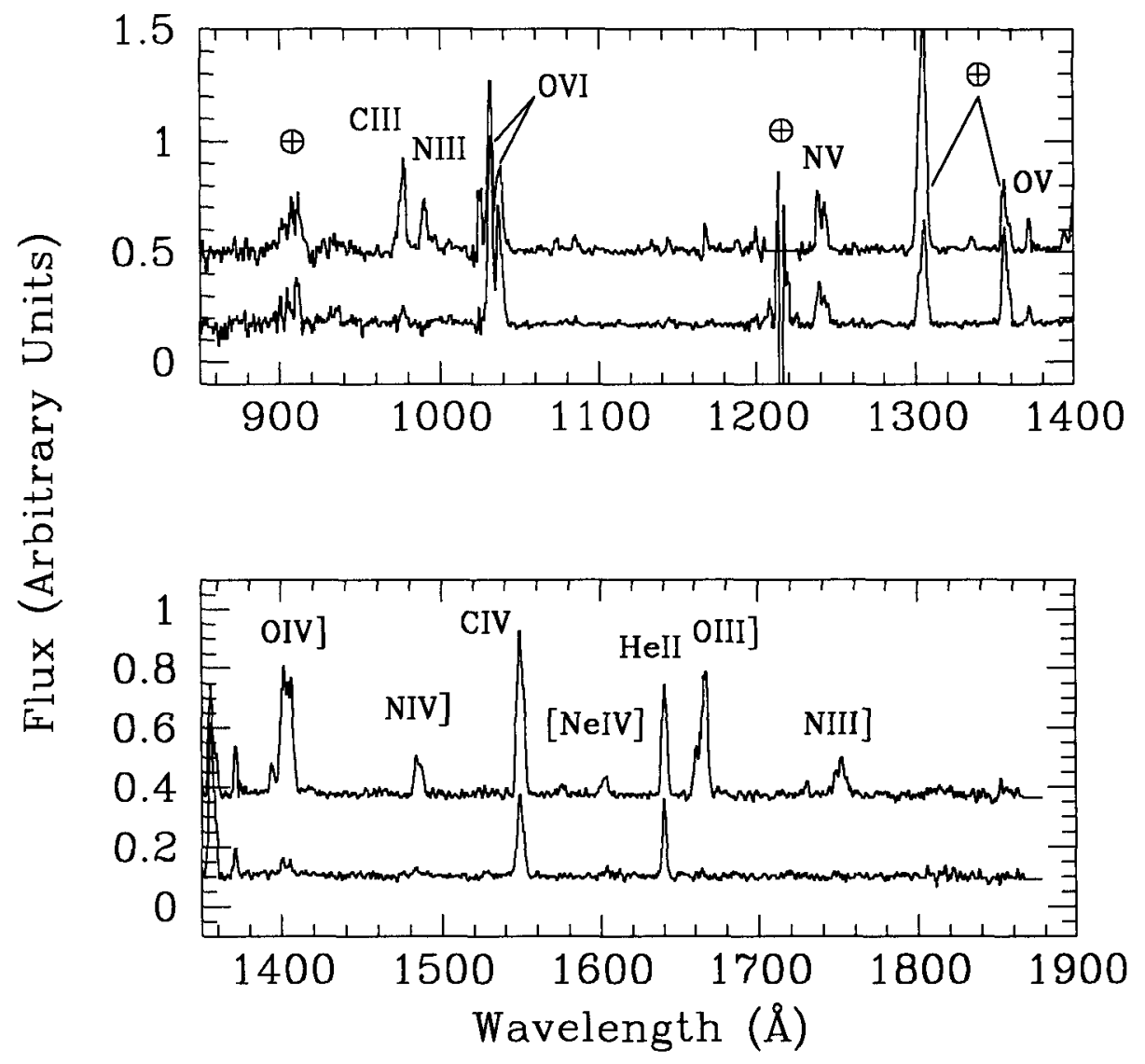

Figure 2. A comparison of HUT spectra of two filaments in the Cygnus Loop. The top line in each panel shows the spectrum of a radiative filament while the bottom line shows a noradiative filament. Strong lines are identified, and and earth symbol denotes regions affected by airglow emissions. Only the highest ionization lines are present at the nonradiative position.

report a spectrum very similar to the high ionization portion of the HUT radiative filament mentioned above, but with much weaker intermediate and low ionization lines (see Figure 1.2). These authors estimate a shock velocity of about $180 \mathrm{~km} \mathrm{~s}^{-1}$ from the HUT data. This filament represents a very recent encounter between the blast wave and an interstellar cloud, and may represent an earlier stage of the evolution that is taking place at the HUT radiative position. Long et al. (1992) find some support for the idea that this shock is rapidly decelerating as it encounters a density step in the ISM, which is consistent with the velocity width of the filament and the fact that it may already be undergoing the transition from nonradiative to radiative conditions (cf. Hester, Raymond, \& Blair 1993). Long et al. (1992) find that a below solar carbon abundance is necessary to match the spectrum at this position, which could indicate that grain destruction has occurred at the HUT radiative position (which was matched with cosmic abundances).

Other HUT SNR observations include the Crab Nebula (Blair et al. 1992) and the bright LMC remnant N49 (Vancura et al. 1992; discussed in the next section). Although the HUT observation of the Crab Nebula failed to detect any new emission lines in the sub-Ly $\alpha$ region, it did detect variations of the C IV:He II ratio in the Crab for the first 
time. Interpretation of these variations is model dependent, but fairly extreme carbon abundances and abundance variations are allowed by the models. This sheds a different light on the possible precursors of this unique object. The relatively normal carbon abundance inferred previously from IUE observations (Davidson et al. 1982) has been used to constrain the mass range of the precursor to 8-10 $M_{\odot}$ (cf. Nomoto et al. 1982). A higher carbon abundance would permit the precursor mass to be above $10 M_{\odot}$.

\section{IUE/HST Results}

The IUE spacecraft has continued to demonstrate its versatility for SNR/ISM observations over the years, and is largely responsible for many of the advances in our understanding of interstellar shocks. As our understanding has improved, the observations have become more comprehensive, combining optical spectroscopy and imagery and/or $\mathrm{X}$-ray data with the IUE spectra to better determine physical conditions and constrain models. Raymond et al. (1988) performed such a study of the "spur" filament in the eastern Cygnus Loop, inferring the geometry and physical parameters in unprecedented detail and demonstrating for the first time that thermal support was not the dominant source of pressure in this filament.

Recently Hester, Raymond, \& Blair (1993) have published a similar tome on the NE nonradiative filaments in the Cygnus Loop, inferring that the blast wave in this region is decelerating and finding that this entire region is on the verge of becoming radiative. Also, Raymond, Wallerstein, \& Balick (1991) have used high dispersion IUE and optical data of a star behind the Vela SNR in conjunction with low resolution IUE spectra of nearby Vela filaments to provide the strongest evidence to date of thermal instabilities in fast shocks predicted theoretically (cf. Innes 1992; Gaetz, Edgar, \& Chevalier 1988).

IUE and optical data have also provided a powerful tool for investigating a few bright extragalactic SNRs. Vancura et al. (1992a,b) performed an extensive analysis of N49 in the LMC, including a HUT observation that detected the O VI lines. By investigating the spatial variations in optical/UV line intensities, it is clear that a wide range of shock velocities are present, indicating a complex preshock medium of relatively high density (to account for the optical and UV luminosity).

Oxygen-rich SNRs are thought to arise from the explosions of massive stars (Type II or Type Ib SNe). Unfortunately, the galactic examples of this class are heavily reddened, which negates UV observations. We have recently obtained IUE spectra of two positions in the LMC remnant N132D (Blair, Raymond, \& Long 1993), which show strong lines of carbon, oxygen, neon, magnesium, and at one position silicon and helium (the first detection of these elements in an oxygen-rich SNR). This combination of elements may indicate significant mixing of the ejecta have taken place in this object. General characteristics of the spectrum, including especially the relative strength of the UV and optical emission lines, point toward photoionization as the dominant emission mechanism as opposed to shock heating, which is similar to the conclusion reached for E0102-7219, the oxygen-rich SNR in the SMC (Blair et al. 1989). Even so, model fits have been unacceptable, partially because emission from various knots gets blended in the IUE spectra.

The power of the Hubble Space Telescope has yet to be turned effectively toward many SNRs. HST is not a very efficient telescope for faint, extended sources, but there are certainly places where it can make significant contributions to our understanding. We have requested time to observe N132D to resolve the structures blended in the IUE spectra and to obtain accurate relative UV/optical line intensities at the same positions; this will greatly improve the comparison to models. It is somewhat frightening to realize 


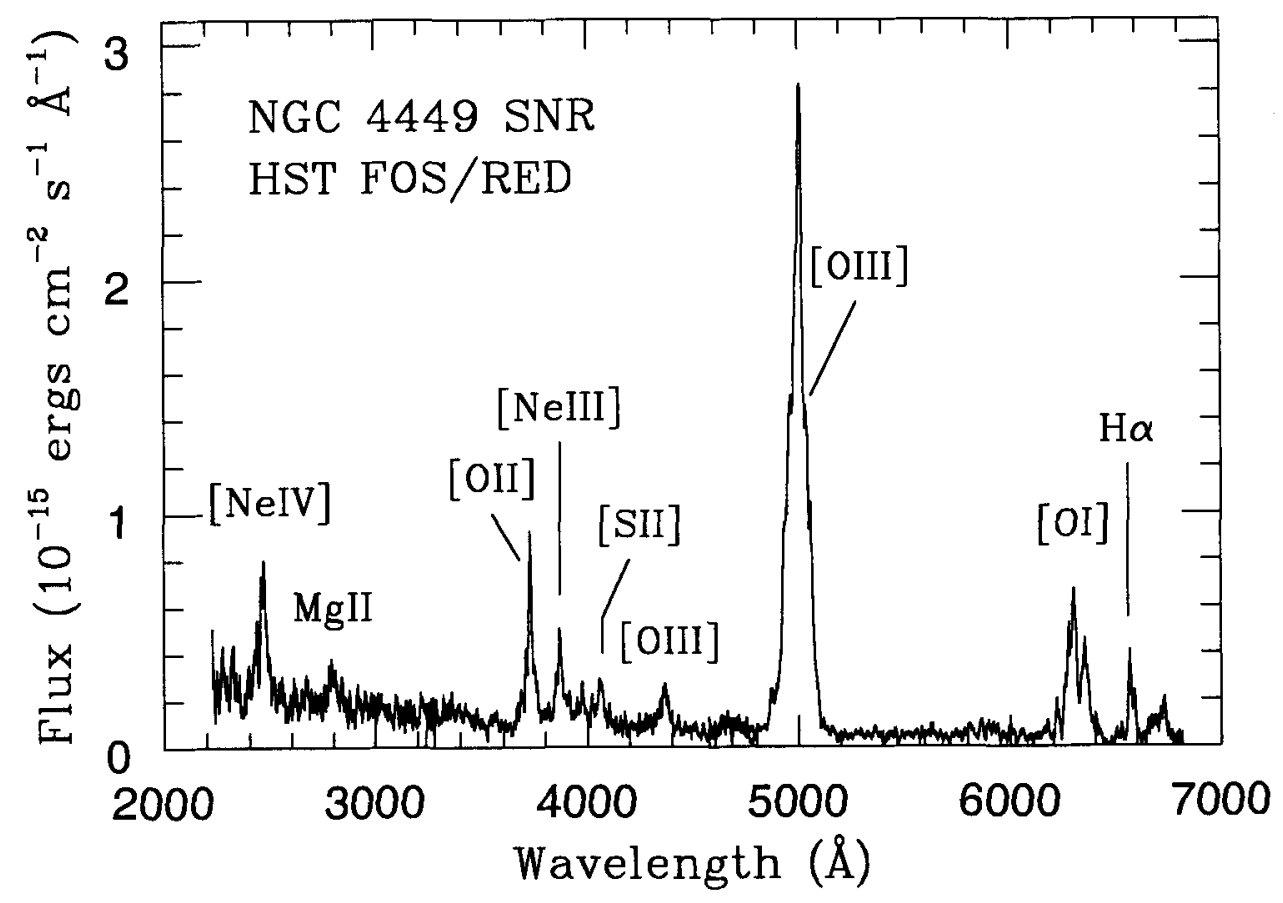

FIGURE 3. A collage of HST/FOS red side spectra of the O-rich SNR in NGC 4449. None of the lines below $3600 \AA$ have been seen previously, and many of the longer wavelength lines are seen more clearly than in ground-based data.The data have been smoothed and combined for presentation here.

how few SNRs have actually been observed successfully in the ultraviolet. Extragalactic SNRs are often better UV targets than galactic SNRs, and HST/FOS has the sensitivity to expand the sample. Figure 1.3 shows FOS/RED spectra of the oxygen-rich remnant in the irregular galaxy NGC 4449 from an FOS/GTO observation. This object is $5 \mathrm{Mpc}$ away and yet is readily detected in reasonable exposure times with FOS. New lines of neon, magnesium, and carbon have been detected in this object for the first time, which should lead to improved estimates of the mass of the precursor star (cf. Blair et al. 1984).

\section{Acknowledgements}

Special thanks go to Jay Holberg for continued assistance with Voyager data reductions, and to the many scientists, engineers, and technical staff people at Johns Hopkins and NASA who worked hard to make HUT and Astro-1 a success. I would like to thank my collaborators for their sound advice and continued support on various aspects of these projects. This work has been supported by the Center for Astrophysical Sciences at the Johns Hopkins University and through various NASA grants and contracts, including NAG 5-1276, NAG 5-1793, NAG 5-1630, NAS 5-27000, and NAS 5-29293. 


\section{REFERENCES}

Blair, W. P., Long, K. S., Vancura, O., \& Holberg, J. B. 1991a, $A p J, 374,202$.

Blair, W. P., et al. 1991b, ApJ, 379, L33.

Blair, W. P., et al. 1992, ApJ, 399, 611.

Blair, W. P., Raymond, J. C., Danziger, I. J., \& Matteucci, F. 1989, ApJ, 338, 812.

Blair, W. P., Raymond, J. C., Fesen, R. A. \& Gull, T. R. 1984, ApJ, 279, 708.

Blair, W. P., Raymond, J. C., \& Long, K. S. 1993, ApJ, 423, 334.

Blair, W. P., Vancura, O., \& Long, K. S. 1993, $A p J L$, submitted.

Broadfoot, L., et al. 1981, J. Geo. Res., 86, 8259.

Cornett, R. H., et al. 1992, ApJ, 395, L9.

Davidsen, A. F., et al. 1992, $A p J, \mathbf{3 9 2}, 264$.

Davidson, K., et al. 1982, ApJ, 253, 696.

Fesen, R. A., Gull, T. R., \& Ketelson, D. A. 1983, ApJS, 51, 337.

Gaetz, T. J., Edgar, R. J., \& Chevalier, R. A. 1988, ApJ, 329, 927.

Hester, J. J., Raymond, J. C., \& Blair, W. P. 1993, $A p J, 420,721$.

Innes, D. E. 1992, $A \& A, \mathbf{2 5 6}, 660$.

Ku, W. H.-M., Kahn, S. M., Pisarski, R., \& Long, K. S. 1984, ApJ, 278, 615.

Long, K. S., Blair, W. P., Vancura, O., Bowers, C. W., Davidsen, A. F., \& Raymond, J. C. 1992, ApJ, 400, 214.

Nomoto, K., et al. 1982, Nature, 299, 803.

Rasmussen, A., \& Martin, C. 1992, ApJ, 396, L103.

Raymond, J. C., Blair, W. P., Fesen, R. A., \& Gull, T. R. 1983, ApJ, 275, 636.

Raymond, J. C., et al. 1988, $A p J$, 324, 869.

Raymond, J. C., Wallerstein, G., \& Balick, B. 1991, ApJ, 383, 226.

Vancura, O., Blair, W. P., Long, K. S., \& Raymond, J. C. 1992a, ApJ, 394, 158.

Vancura, O., et al. 1992b, $A p J, \mathbf{4 0 1}, 220$.

Vancura, O., Blair, W. P., Long, K. S., Raymond, J. C., \& Holberg, J. B. 1993, ApJ, 417, 663 